\title{
Experiencia grupal breve para pacientes con trastornos de ansiedad en un Centro de Salud Mental.
}

Brief group experience for patients with auxiety disorders in a Mental Health Center.

\author{
Silvia Poves Oñate ${ }^{\mathrm{a}}$, Rafael Romero Gamero ${ }^{\mathrm{b}}$, Nicolás Vucínovich ${ }^{\mathrm{c}}$.
}

${ }^{a}$ Psicóloga Clínica. Centro de Salud Mental de Aranjuez. Orden Hospitalaria San Juan de Dios. Madrid. ${ }^{b}$ Psicólogo Clínico. Unidad de Salud Mental Comunitaria-Oriente. Hospitales Universitarios "Virgen del Rocio". Sevilla. "Psiquiatra. Unidad de Salud Mental Comunitaria-Oriente. Hospitales Universitarios "Virgen del Rocío". Sevilla.

Correspondencia: Silvia Poves Oñate (sppoves@yahoo.es)

Recibido: 16/09/2009; aceptado: 2/10/2009

RESUMEN: Introducción: Los trastornos de ansiedad son uno de los diagnósticos más frecuentes en la población general. En este estudio describimos una experiencia de tratamiento psicológico grupal para pacientes con trastornos de ansiedad, especialmente Trastorno de Ansiedad Generalizada.

Método: Nuestro método consiste en el desarrollo de un grupo terapéutico, con frecuencia quincenal y 12 sesiones de duración. En él se trabaja explícitamente el afrontamiento activo de las situaciones generadoras de ansiedad, favoreciendo la adquisición de nuevos recursos psicológicos y tomando en cuenta los aspectos relacionales involucrados en el mantenimiento del cuadro.

Resultados: Los resultados son prometedores tomando como criterio de eficacia el alta clínica y la recuperación del nivel de actividad habitual lo que nos anima a seguir trabajando en esta modalidad de intervención desde el Sistema Sanitario Público.

PALABRAS CLAVE: Trastorno de ansiedad, Psicoterapia Grupal, Recursos de Afrontamiento.
ABSTRACT: Introduction: Anxiety disorders are one of the more prevalent in general population. This study reports one group experience targeting patients with anxiety disorders, mainly on Generalized Anxiety Disorder.

Method: Group psychotherapy described is developed through 12 biweekly sessions. Treatment focuses on active coping of stress events, encouraging the purchase of new psychological resources and considering the relational aspects involved in the maintenance of the disorder.

Results: Results are promising considering discharge after the intervention and recovery of normal activity. Data support continuing this modality of intervention in our public health system.

KEYWORDS: Anxiety Disorder, Group psychotherapy, Coping Skills. 


\section{Introducción}

Los trastornos de ansiedad son, junto a los trastornos depresivos, los diagnósticos psicológicos más frecuentes en la población general. Entre ellos el Trastorno de Ansiedad Generalizada (T.A.G. en adelante) es uno de los más frecuentes con una prevalencia vital que llega al 5,4\% (1). Por otro lado, la comorbilidad tanto con diagnósticos del eje I y eje II es la regla más que la excepción, con datos del $90 \%$ (2) para alteraciones emocionales de tipo ansioso-depresivo, y del $50 \%$ para trastornos de la personalidad del grupo C. Su inicio suele estar sobre la primera juventud y el curso supone ser crónico en gran parte de los diagnosticados. Esto ha llevado a muchos autores a considerar el T.A.G como un trastorno de personalidad ansiosa (3).

Los pacientes afectados de un T.A.G. se caracterizan por "ansiedad generalizada y persistente, que no está limitada y ni siquiera predomina en ninguna circunstancia ambiental en particular (es decir, se trata de una "angustia libre flotante")" (4). La ansiedad se manifiesta en los tres ejes de respuesta descritos por P. J. Lang en 1968 (5) (psicológico, fisiológico - emocional y motor) pero resulta especialmente característica la clínica en torno a la preocupación excesiva, crónica, irrealista y no controlable (6). Asimismo son frecuentes síntomas de hiperactividad vegetativa como manos frías y pegajosas, boca seca, sudoración, náuseas, diarreas, problemas para tragar o sensación de que se tiene algo en la garganta y tensión muscular, además de respuestas de sobresalto exageradas y síntomas depresivos. El curso característico es fluctuante y crónico de tal modo que suelen estar presentes estresores crónicos así como los empeoramientos clínicos se asocian a la presencia de acontecimientos vitales estresantes. Desde una perspectiva cognitiva, podríamos decir que estos pacientes poseen escasas habilidades de afrontamiento de los cambios desarrollando un estilo cognitivo ansioso según el cual cada estímulo es interpretado como amenazante o que sobrepasa los recursos propios para hacerles frente (7).

En general, la importancia social de este trastorno es elevada, ya que algunas investigaciones muestran que alrededor del $33-40 \%$ de la población puede necesitar tratamiento debido a sus problemas de ansiedad (8), tal como indica una trabajo de Fifer (9), en el que apareció que un 33\% de los pacientes evaluados (6.000) de las consultas médicas de organizaciones importantes en EE.UU. mostraron fuertes síntomas de ansiedad o de trastornos de ansiedad.

Los resultados sobre tratamientos indican una eficacia semejante entre terapias farmacológicas con antidepresivos y terapias cognitivo-conductuales y superior de ésta última respecto al uso exclusivo de benzodiazepinas en fase aguda. Se observa, eso sí, una clara superioridad del tratamiento psicológico respecto al farmacológico a medio y largo plazo (10). 
ORIGINALES Y REVISIONES

En los Centros de salud mental suele ser frecuente encontrar pacientes aquejados por este trastorno, sin embargo, la alta comorbilidad comentada puede llevarnos a pasar por alto su existencia o minimizarla con las posibles repercusiones negativas en la respuesta a los tratamientos. Por consiguiente un diagnóstico correcto unido a un trabajo explicito de éste trastorno a través de una intervención directa se plantea como prioritaria al trabajar con pacientes con patología de la esfera ansioso-depresiva. Consideramos que la aplicación de un tratamiento con apoyo empírico como es la terapia cognitivo-conductual (TCC en adelante) en formato grupal supone, no solo que los pacientes reciban un tratamiento efectivo, sino también eficiente, aspecto éste nada despreciable si pretendemos maximizar los recursos terapéuticos disponibles en la asistencia sanitaria publica. Dicho tratamiento tiene como característica principal su carácter multicomponente, fundamental en el abordaje de los trastornos de ansiedad, para tratar las dificultades cognitivas, fisiológicas y emocionales que conllevan. Por otro lado, el tratamiento grupal cuenta con numerosas investigaciones que avalan su eficacia, puesto que es una herramienta terapéutica muy poderosa y rentable que fomenta las interacciones interpersonales (11).

Presentamos a continuación la experiencia de terapia de grupo llevada a cabo en un Centro de Salud Mental con un grupo de mujeres aquejadas de trastornos de ansiedad, siendo el diagnóstico más común el de Trastorno de Ansiedad Generalizada.

\section{Metodología}

Muestra:

La muestra está compuesta por 13 mujeres que acuden al CSM-Aranjuez de forma periódica y con un diagnóstico genérico de trastorno de ansiedad. En concreto, el diagnóstico principal de TAG en 10 de ellas de las cuales, 4 presentan comorbilidad con trastornos de la esfera depresiva, incluyendo trastornos adaptativos. La media de edad es 34.08 años (desviación típica: 7.93) y en su mayoría trabajan $(84,6 \%)$. De ellas, un $63,6 \%$ no podían desempeñar su actividad laboral a consecuencia de su trastorno. El motivo principal de derivación a la intervención grupal era la escasa respuesta a las intervenciones farmacológicas, tanto a nivel de atención primaria como de especializada, y la motivación al tratamiento psicológico grupal. Se trata de un grupo heterogéneo en cuanto a gravedad de los síntomas y sin embargo más homogéneo en cuanto a características personales y relacionales que favorecieran su identificación. Más allá de los diagnósticos, la topografía de los síntomas también era heterogénea y las expectativas de cambio 
Tabla 1

\begin{tabular}{|l|c|c|c|}
\hline \multicolumn{1}{|c|}{ DIAGNÓSTICOS } & EDAD & NIVEL DE ESTUDIOS & ACTIVIDAD LABORAL \\
\hline $\begin{array}{l}\text { T. Adaptativo } \\
\text { Conflictos laborales }\end{array}$ & 23 & Educación Obligatoria & Si ILT \\
\hline $\begin{array}{l}\text { TAG } \\
\text { T. Depresivo }\end{array}$ & 32 & Sin estudios & Si \\
\hline $\begin{array}{l}\text { TAG } \\
\text { Fobia específica }\end{array}$ & 42 & Educación Obligatoria & \\
\hline TAG & 25 & & Si ILT \\
\hline $\begin{array}{l}\text { TAG } \\
\text { Conflictos laborales }\end{array}$ & 26 & Bachillerato & Si ILT \\
\hline T. pánico & 43 & Sin estudios & Si \\
\hline Fobia social & 26 & Universitaria & Si ILT \\
\hline TAG & 42 & Educación Obligatoria & No \\
\hline TAG & 31 & Universitaria & Si \\
\hline TAG & 34 & Formación profesional & No \\
\hline TAG & 45 & Educación Obligatoria & Si \\
\hline $\begin{array}{l}\text { TAG } \\
\text { Distimia }\end{array}$ & 45 & Educación Obligatoria & Si ILT \\
\hline TAG & 29 & Formación profesional & \\
\hline
\end{tabular}

aún más. En la elección de la muestra se ha eliminado, sin embargo, la variabilidad en cuanto al género de las participantes buscando una mayor cohesión grupal y favorecer el aprendizaje vicario (12). Todas consultaron en nuestro servicio por problemas de ansiedad que surgen de forma inesperada en la historia del individuo y que cursan con deterioro social, laboral o de otras áreas importantes en su desarrollo vital, tal como se describió más arriba. De hecho, muchas de ellas se encuentran en situación de incapacidad laboral temporal y expresan dificultades de relación en su contexto socio - familiar que en algunos casos generan sentimientos de desesperanza y falta de motivación que agravan el cuadro.

\section{Procedimiento}

La terapia grupal estuvo compuesta de 12 sesiones con una frecuencia quincenal, con un número de 6-7 miembros por grupo en torno a una tarea explícita, que en todos los casos, es la detección y el afrontamiento activo de las situaciones en las que aparece la respuesta de ansiedad. Se trataba de grupos cerrados con un encuadre del que se informaba en entrevistas de valoración individual de la idoneidad de la terapia grupal.

De esta forma, los objetivos a los que se dirige el grupo de ansiedad son los siguientes: 
ORIGINALES Y REVISIONES

- Aumentar el conocimiento de la respuesta de ansiedad en su vertiente fisiológica, emocional, cognitiva y motora.

- Comprender los mecanismos de aparición y mantenimiento de los síntomas asociados a la ansiedad.

- Aumentar el autoconocimiento de forma que se relacione la sintomatología con las circunstancias vitales por las que atraviesa la persona y de esta forma se integre la experiencia vivida dentro de la historia personal.

- Disminuir el nivel de activación (arousal) mediante el aprendizaje y puesta en práctica de técnicas como respiración abdominal y relajación muscular.

- Detectar y corregir creencias irracionales que juegan un importante papel en el mantenimiento del trastorno.

- Activar a la persona para la búsqueda de soluciones alternativas y flexibles mediante el entrenamiento en resolución de problemas de forma que supere la inhibición conductual y la evitación.

- Afrontar activamente las situaciones generadoras de ansiedad haciendo especial hincapié en las relaciones interpersonales, favoreciendo la aparición de conductas asertivas.

- Favorecer la adquisición de una visión sobre sí mismos, los demás y el mundo objetiva y flexible.

En cada sesión se recogía por parte de la terapeuta las verbalizaciones de las integrantes del grupo así como sus reacciones frente a las demás participantes. Se reflejaban los contenidos tratados y el clima emocional del grupo, que posteriormente analizaremos de modo más descriptivo.

Nuestra premisa de partida es que la ansiedad es una respuesta adaptativa y automática del individuo que surge cuando éste se encuentra en una situación evaluada como peligrosa (de forma real o imaginaria) o que supera sus recursos personales (13).

Distinguimos tres fases en la adquisición de dichos recursos para el abordaje de la sintomatología ansiosa, de forma análoga al entrenamiento en inoculación de estrés según el modelo de Meichembaum (14). Estas fases nos han servido como un esquema general y suficientemente flexible que ha constituido el "marco" del grupo terapéutico:

- Fase educativa: adquisición de un marco conceptual que permita al paciente comprender cómo puede afectarle el estrés y cómo puede hacerle frente. Nos apoyamos en material educativo en formato escrito sobre el que las participantes pudieran trabajar entre sesiones.

- Fase de adquisición de habilidades: entrenamiento en estrategias de afrontamiento dirigidas a reducir la activación, exponerse a las situaciones generadoras de ansiedad, solucionar problemas interpersonales,... También se hace hincapié en la construcción de una nueva narrativa personal que permita al individuo 
asimilar la experiencia vivida de forma adaptativa, integrando lo sucedido en su forma de percibir el mundo, dotando de significado lo ocurrido y las emociones experimentadas.

- Fase de consolidación: poner en práctica las habilidades adquiridas y entrenadas. En esta fase, nos centramos en el "afrontamiento" de la ansiedad.

La respuesta de ansiedad genera en muchas ocasiones conductas evitativas que llevan a la persona a no afrontar o escapar de situaciones que ellos evalúan como peligrosas con lo que su percepción de no ser capaces de afrontarlas aumenta. Todo ello genera una sensación de falta de control sobre los acontecimientos que comienza a ser uno de los ejes de su autoconcepto y va minando de forma progresiva su autoestima, que hasta entonces no era considerada fuente de malestar.

\section{Resultados:}

Comentamos los datos de dos convocatorias grupales que han recibido el procedimiento explicado y con la misma terapeuta. Del total de las 13 pacientes, 9 han recibido tras la conclusión del grupo el alta por mejoría (69.2\%), 1 ha abandonado (7.7\%) tras la cuarta sesión y 3 siguen acudiendo a las citas programadas $(23.1 \%)$. No se han detectado recaídas en las 9 primeras durante un año después de la conclusión de las sesiones grupales, tomando como criterio de recaída acudir de nuevo a consulta. De las 3 pacientes que continúan necesitando tratamiento psicológico tras un año del fin del grupo terapéutico programado, destaca en una de ellas la comorbilidad con un trastorno de la esfera depresiva (distimia) y en otra, acontecimientos vitales estresantes posteriores a la finalización del grupo que actuaron como desencadenantes de un episodio ansioso - depresivo posterior.

En cuanto a calidad de vida y nivel de actividad, el $85,71 \%$ recuperaron su nivel de actividad cotidiano: de las 7 pacientes con actividad laboral fuera del hogar en situación de Incapacidad Laboral Transitoria al inicio del grupo todas, excepto una que continuaba en tratamiento tras un año, realizaban su trabajo con normalidad tras el alta terapéutica o al cabo de unos meses (ver tabla 2).

Tabla 2

\begin{tabular}{|c|c|c|c|}
\hline $\mathrm{N}^{\circ}$ ALTAS & $\mathrm{N}^{\circ}$ ABANDONOS & $\%$ ALTAS & $\mathrm{N}^{\circ}$ ILT al alta \\
\hline 9 & 1 & $69,2 \%$ & 1 \\
\hline
\end{tabular}


ORIGINALES Y REVISIONES

Mas allá de los resultados descritos sobre número de altas y calidad de vida en cada una de las participantes, es objeto de este estudio analizar de forma descriptiva los resultados obtenidos en cuanto a los objetivos propuestos y la vivencia subjetiva de las pacientes. Para ello, trataremos de describir las verbalizaciones referidas por las participantes que resulten relevantes a la hora de determinar los contenidos/variables del grupo que han resultado subjetivamente más importantes.

Entre los temas y objetivos en los que están interesadas para tratar en el grupo, destacan los siguientes:

- Búsqueda de apoyo social: "Conocer las experiencias de los otros", "Buscar amigos q me apoyen", "Necesito hablar".

- Dificultades relacionales: "Los demás creen que soy más débil", "Me cuesta pedir ayuda", "Mi familia no me comprende", "No es justo que los demás sufran mis reacciones"

- Dificultades de asertividad: "Defender mis derechos en el trabajo", "Ser más yo", "No sé delegar", "Absorbo los problemas de los demás"

- Sintomatología: "Que desaparezca la ansiedad", "Estar menos irritable", "Afrontar las situaciones que me dan miedo", "Noto falta de control".

Siguiendo la propia estructura del grupo, se observa mayor hincapié en la sintomatología a lo largo de las primeras sesiones, coincidiendo con la primera fase psicoeducativa. Desde el principio, la intensidad emocional era elevada en ambos grupos y el clima grupal así como el encuadre favorecían la expresión emocional. A medida que avanzaba el grupo, comenzaron las autorrevelaciones (alrededor de la $5^{\text {a }}$ sesión) y se observaba cómo las técnicas y habilidades de afrontamiento de la ansiedad y resolución de problemas eran utilizadas por las participantes como medio para ilustrar sus vivencias, poniendo siempre ejemplos de lo sucedido en sus vidas. Hubo materiales que utilizaron (material de psicoeducación de ansiedad, material autoayuda asertividad) y otros que fueron propuestos por la terapeuta pero que el grupo no adoptó como propios (autorregistro). En las últimas sesiones $\left(10^{\mathrm{a}}-12^{\mathrm{a}}\right.$ sesión) apenas comentaban síntomas sino que se centraban en sus vivencias personales y en su capacidad para cambiarlas.

\section{Discusión}

Es de destacar como principal resultado que casi el $70 \%$ de las pacientes se encontraban, tras recibir un programa de terapia grupal breve y con objetivos específicos, en situación de continuar su vida sin precisar tratamiento especializado por nuestra parte, como se observa a través de las altas recibidas. Consi- 
deramos particularmente relevante este dato si nos atenemos a la tendencia a la cronicidad que se observa en estos trastornos. El alta no supone solo una mejoría de la sintomatología propia de estos cuadros si no un cambio de actitud ante su padecimiento, responsabilizándose ellas en su resolución al poner en práctica los recursos para manejar los conflictos aprendidos en la experiencia grupal. Lo anterior supone un incremento en su nivel de autoeficacia con la correspondiente mejora en su autoconcepto (15).

El encuadre grupal favorece la percepción de sus propias dificultades al ser expresadas por otra persona con la que no mantienen vinculación emocional lo que les va a permitir comprenderse mejor. Otra consecuencia directa del abordaje en grupo de esta patología consiste en afrontar situaciones sociales probablemente alejadas dadas las conductas evitativas que aparecen. Por otro lado, el apoyo social favorece una mejoría en el estado de ánimo que en muchas ocasiones está muy disminuido por la incomprensión que perciben por parte de sus personas cercanas. En la situación segura de grupo terapéutico, el paciente irá afrontando situaciones que puede generalizar en su vida diaria a la par que adquiere recursos de afrontamiento de forma activa que tendrá que poner el práctica fuera del grupo.

Este aspecto entra en relación con el uso que los pacientes hacen de los servicios sanitarios. Un papel más activo en su trastorno favorece la puesta en marcha de recursos personales y sociales en detrimento de los sanitarios que suponen una estrategia más pasiva.

En las experiencias grupales descritas constatamos que, tras un año de su conclusión, ninguna de las pacientes que recibió el alta ha vuelto a solicitar asistencia. Los resultados nos animan a continuar en esta línea de intervención debido a la mejora general a largo plazo que han disfrutado la mayoría de las participantes. En este sentido, analizando el perfil de las pacientes que continúan acudiendo a tratamiento al Centro de Salud Mental, es destacable el hecho de todas ellas presentaban comorbilidad con otros trastornos en el momento de su evaluación y derivación al grupo terapéutico. De cara a nuevas experiencias futuras, será necesario decidir, de cara a la selección de pacientes, qué perfil es más adecuado. De este estudio se desprende que el formato de terapia breve grupal desarrollado, es más eficaz en la medida que el cuadro clínico de las pacientes es más concreto y focalizado en la esfera ansiosa. De hecho, la mejoría fue notable también en aquellas pacientes cuyo diagnóstico no era TAG sino Fobia Social o Trastorno de Pánico. Podemos sugerir que en pacientes con cuadros mixtos, la intervención más adecuada puede ser de una duración superior.

Por otro lado, también resulta fundamental trabajar la adherencia a los tratamientos que resulta requisito indispensable de cara a su eficacia. En la situación grupal, el favorecimiento de la cohesión grupal redunda en dicho objetivo así como la adecuada selección de pacientes. Dicha fase del pre - tratamiento debe 
ORIGINALES Y REVISIONES

ser considerada con especial atención por su influencia sobre la motivación y posterior desarrollo del grupo.

En el presente estudio nos decantamos por inclusión de pacientes sólo del género femenino lo que permitió abordar algunas cuestiones relativas a la mujer de tal modo que las cuestiones relacionales estaban muy presentes. Cabe cuestionarse si el contrapunto del género (inclusión de varones) ahonda en la posibilidad de desarrollar diferentes patrones de conducta y perspectivas del problema enriqueciendo los resultados. Consideramos en este caso que la nula variabilidad en cuanto a género favoreció el elevado tono emocional desde el inicio de las sesiones y esto resultó fundamental dada la brevedad del formato grupal implementado.

\section{BIBLIOGRAFÍA:}

(1) Wittchen, H-U, Zhao, S., Kessler, R.C. y EAton, W.W. 1994. DSM - III - R generalized anxiety disorder in the National Comorbidity Survey. Archives of General Psychiatry, 51: 355 - 364.

(2) Dugas, M.J. y Ladoceur, R. 1997. Análisis y tratamiento del trastorno por ansiedad generalizada. En V.E. Caballo (dir): Manual para el tratamiento cognitivo - conductual de los trastornos psicológicos (Vol. 1). Madrid: Siglo XXI.

(3) RAPEE, R.M. 1995. Trastorno por ansiedad generalizada. En V.E. Caballo, G. Buela - Casal y J.A. Carrobles (dirs.): Manual de psicopatología y trastornos psiquiátricos: Vol.1. Madrid: Siglo XXI.

(4) Organización Mundial de la SAlud. Clasificación estadística internacional de enfermedades y problemas relacionados con la salud. - 10a. revisión. Washington, D.C.:OPS; 1995.

(5) LANG, P.J. Fear reduction and fear behavior: Problems in treating a construct. En J.M. Shleien (Ed.). Research in Psychotherapy, III. Washington: American Psychological Association. 1968.

(6) BoRKovec ET AL. The nature of normal and pathological worry. En R.M. Rappee y D.H. Barlow (Eds.), Cronic anxiety: generalized anxiety disorder and mixed anxiety depression (pp. 29 - 51.) Nueva York: Guilford. 1991

(7) BECK, A.T. y EMERY G. Anxiety disorders and phobias: a cognitive perspective. Nueva York: Basic Books. 1985.

(8) BARLow, D.H. Anxiety and its disorders. Nueva York: Guidford. 1988.

(9) Fifer, S.K., Mathias, S.D., Patrick, D.L., Majonson, P.D., LubeCK, D.P., \& Buesching, D.P. Untreated anxiety among adult primary care patients in a health maintenance organization. Archives of General Psychiatry, 51, 740-750. 1994.

(10) Power, K.G., Simpson, R.J., Swanson, V., Wallace, L.A., Feistner, A.T.C., \& Sharp, D. A controlled comparison of cognitive-behaviour therapy, diazepam, and placebo alone and in combination, for the treatment of generalized anxiety disorder. Journal of Anxiety Disorders, 4, 267-292. 1990.

(11) Vinogradov S, Yalom ID. Guía breve de psicoterapia de grupo. Barcelona: Paidós. 1996.

(12) Yalom, I. D. Teoría y Práctica de la Psicoterapia de Grupo. México: Fondo Cultura Económica., 1986. 
(13) LAZARUS, R.S. y FolKman, S. Estrés y procesos cognitivos. Barcelona: Martínez Roca, 1986.

(14) Meichembaum, D. Manual de Inoculación de Estrés. Barcelona: Martínez Roca, 1987.

(15) Bandura, Albert. Social Learning Theory. Englewood Cliffs, New Jersey: Prentice Hall. 1977 